\title{
Conservation of the Hydrogen-bonded Pyridone Homosynthon in Halogen-bonded Cocrystals
}

\author{
Nikola Bedeković, ${ }^{a}$ Luka Fotović, ${ }^{a}$ Vladimir Stilinović, ${ }^{* *}$ Dominik Cinčića ${ }^{*}$ \\ aUniversity of Zagreb, Faculty of Science, Department of Chemistry, Horvatovac 102A, 10000 Zagreb, Croatia; \\ nbedekovic@chem.pmf.hr (N.B.), lfotovic@chem.pmf.hr (L.F.) vstilinovic@chem.pmf.hr (V.S.), \\ dominik@chem.pmf.hr (D.C.) \\ * Correspondence: vstilinovic@chem.pmf.hr (V.S.); Tel.: +385-1-4606-371 (V.S.); dominik@chem.pmf.hr \\ (D.C.); Tel.: +385-1-4606-362 (D.C.)
}

Table of Content

Item

Page

Figures S1-S7 (ORTEP representations of the formula units of the prepared compounds)

Figures S8 (PXRD patterns of the prepared compounds)

Figures S9-S15 (DSC and TG curves of the prepared compounds)

Figure S16-S23 (IR spectra of the prepared compounds)

Table S1 Crystallographic data

Table S2. Halogen bond lengths and their relative shortenings (rs) in obtained compounds. 


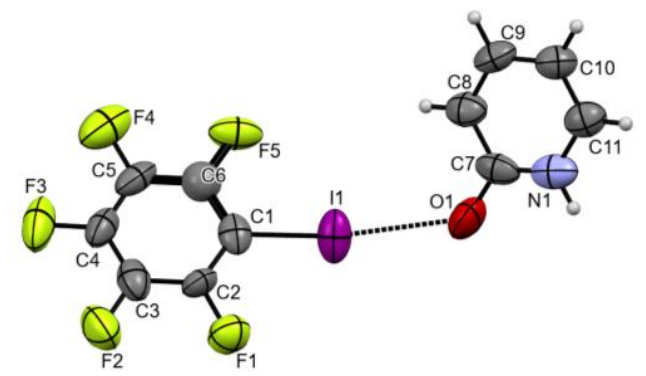

Figure S1. Molecular structure of $\mathbf{b 3 p m}$ showing the atom-labelling scheme. Displacement ellipsoids are drawn at the $50 \%$ probability level, and $\mathrm{H}$ atoms are shown as small spheres of arbitrary radius.

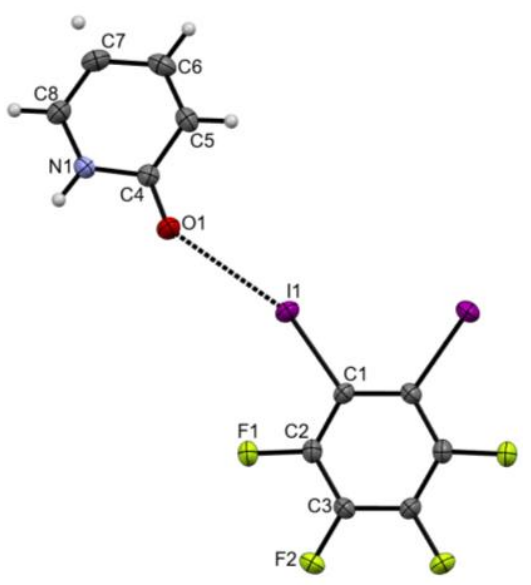

Figure S2. Molecular structure of (b4pm)(13tfib) showing the atom-labelling scheme. Displacement ellipsoids are drawn at the $50 \%$ probability level, and $\mathrm{H}$ atoms are shown as small spheres of arbitrary radius.

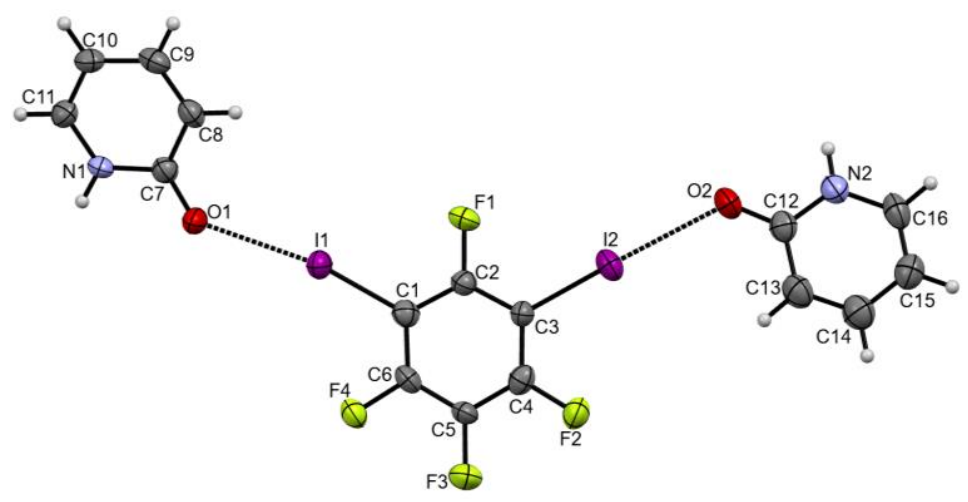

Figure S3. Molecular structure of (b4pm)(13tfib) showing the atom-labelling scheme. Displacement ellipsoids are drawn at the $50 \%$ probability level, and $\mathrm{H}$ atoms are shown as small spheres of arbitrary radius.

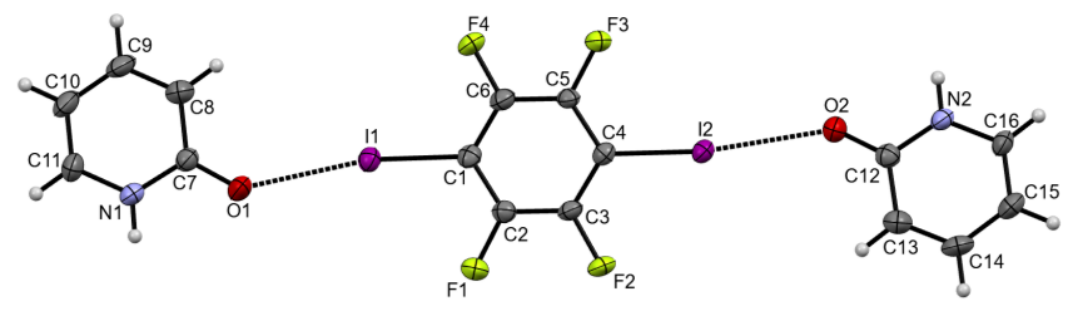

Figure S4. Molecular structure of (b4 pm) 2 (14tfib) showing the atom-labelling scheme. Displacement ellipsoids are drawn at the $50 \%$ probability level, and $\mathrm{H}$ atoms are shown as small spheres of arbitrary radius. 


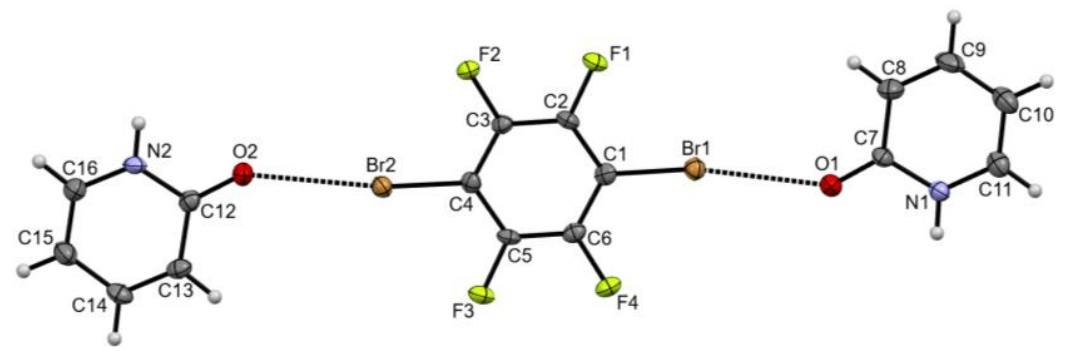

Figure S5. Molecular structure of $(\mathbf{b} 4 \mathbf{p m})(\mathbf{i p f b})$ showing the atom-labelling scheme. Displacement ellipsoids are drawn at the $50 \%$ probability level, and $\mathrm{H}$ atoms are shown as small spheres of arbitrary radius.

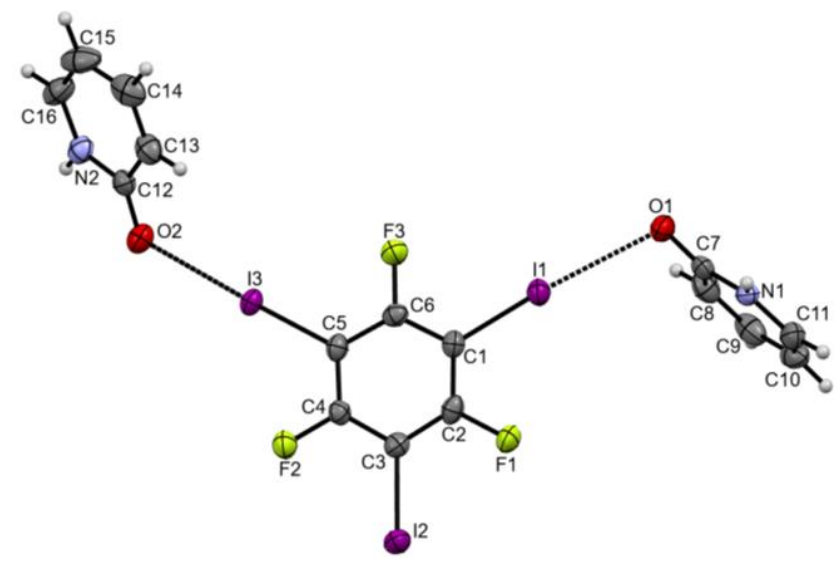

Figure S6. Molecular structure of (b4 pm)(135tfib) showing the atom-labelling scheme. Displacement ellipsoids are drawn at the $50 \%$ probability level, and $\mathrm{H}$ atoms are shown as small spheres of arbitrary radius.

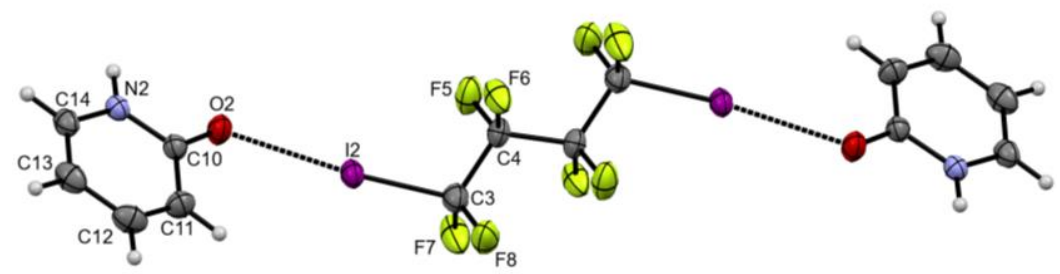

Figure S7. Molecular structure of (b4 pm)(135tfib) showing the atom-labelling scheme. Displacement ellipsoids are drawn at the $50 \%$ probability level, and $\mathrm{H}$ atoms are shown as small spheres of arbitrary radius. 


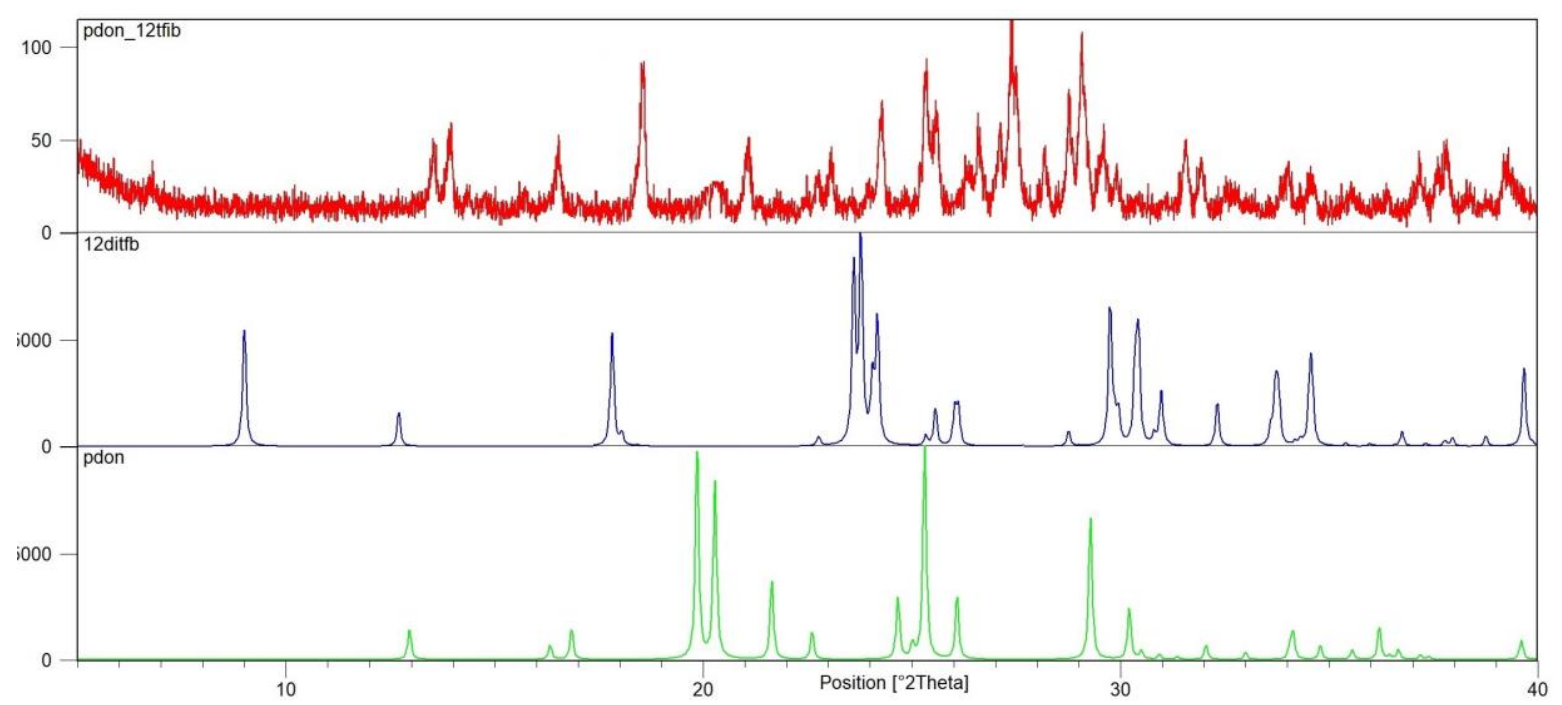

Figure S8. Comparison of the PXRD patterns of (12ditfb)(pdon)2, 12ditfb and pdon.

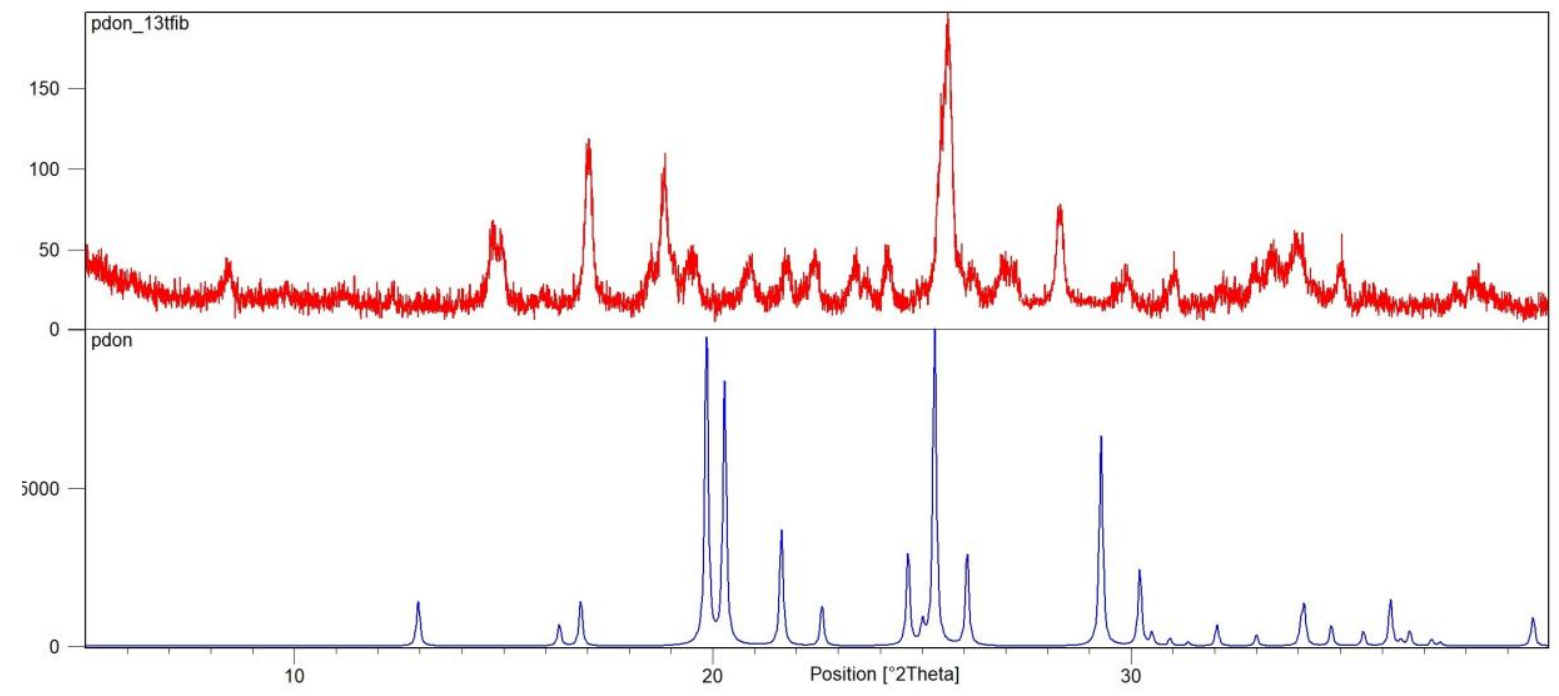

Figure S9. Comparison of the PXRD patterns of (13ditfb)(pdon)2 and pdon. 


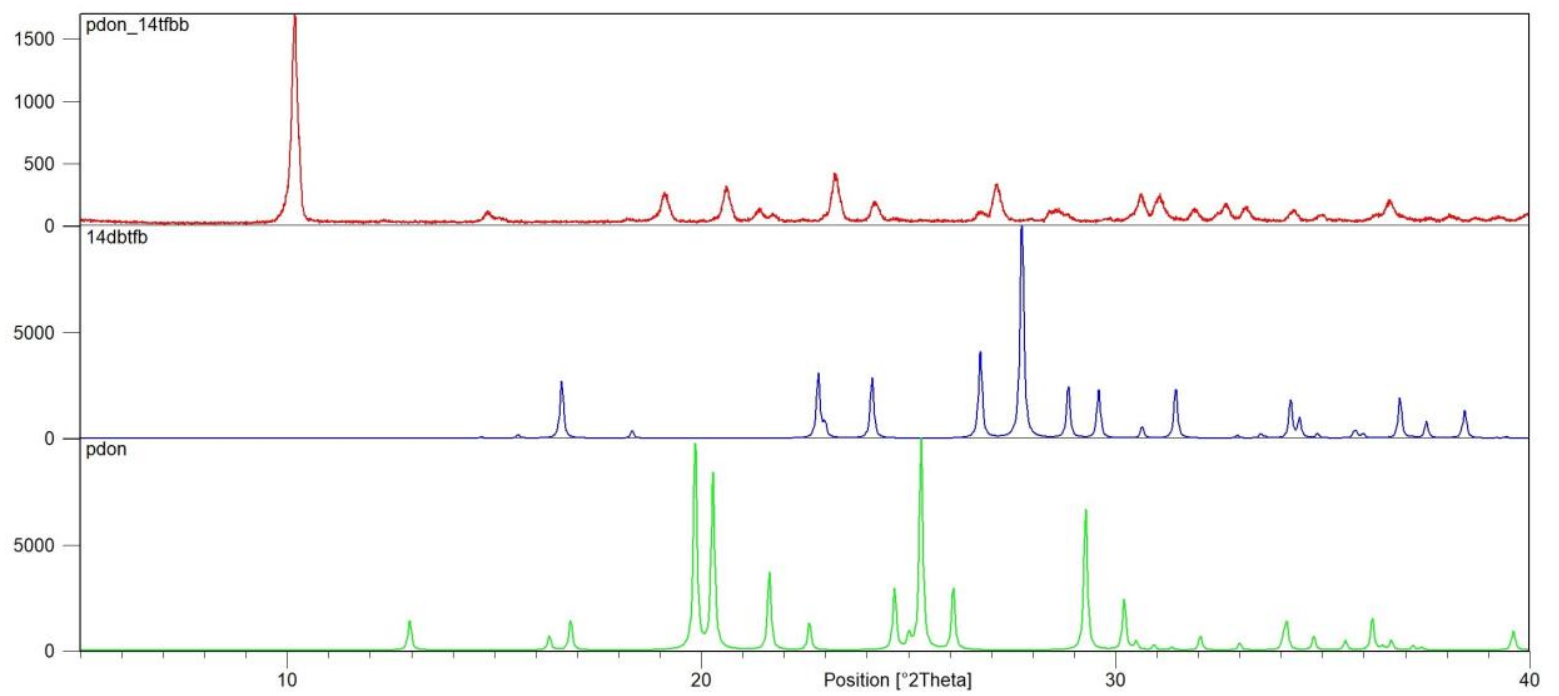

Figure S10. Comparison of the PXRD patterns of (14dbtfb)(pdon)2, 14dbtfb and pdon.

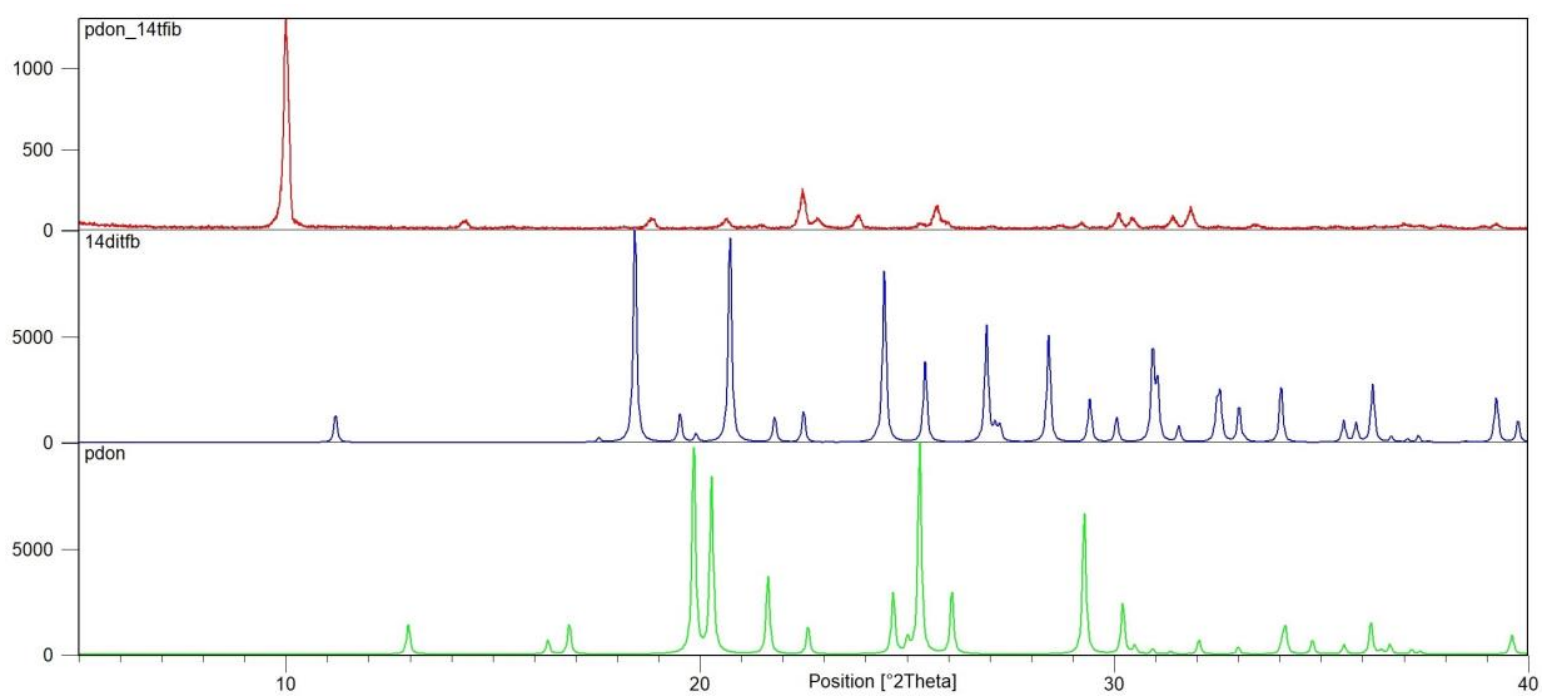

Figure S11. Comparison of the PXRD patterns of (14ditfb)(pdon)2, 14ditfb and pdon. 


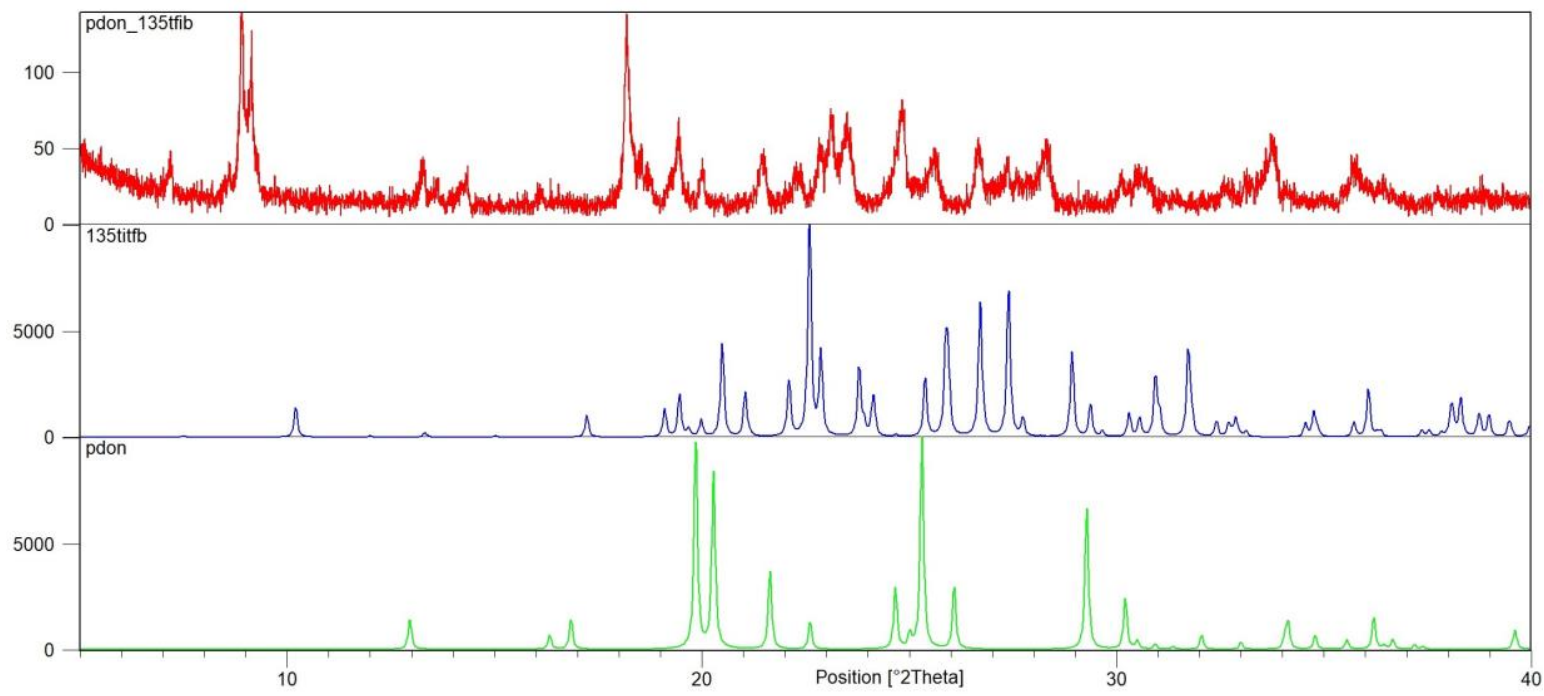

Figure S12. Comparison of the PXRD patterns of (135titfb)(pdon)2, 135titfb and pdon.

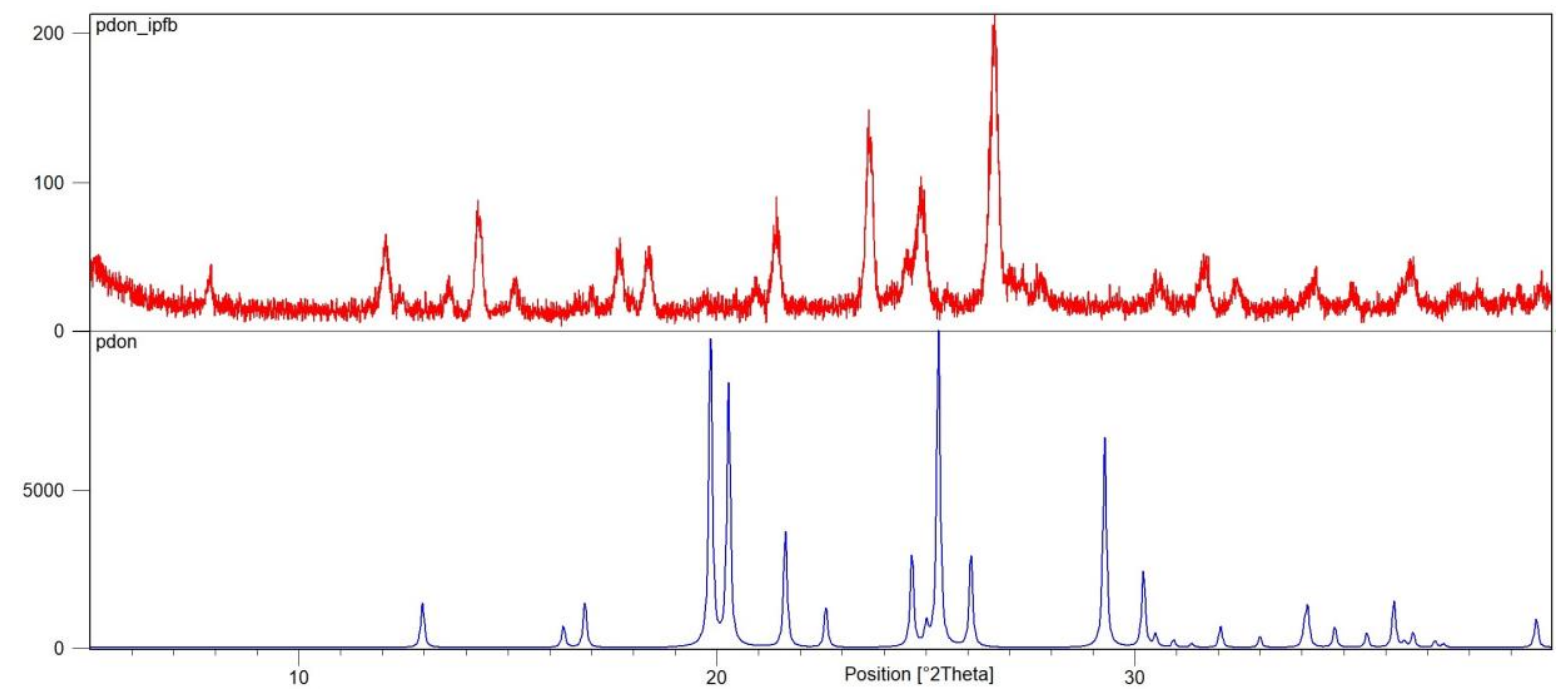

Figure S13. Comparison of the PXRD patterns of (ipfb)(pdon) and pdon. 


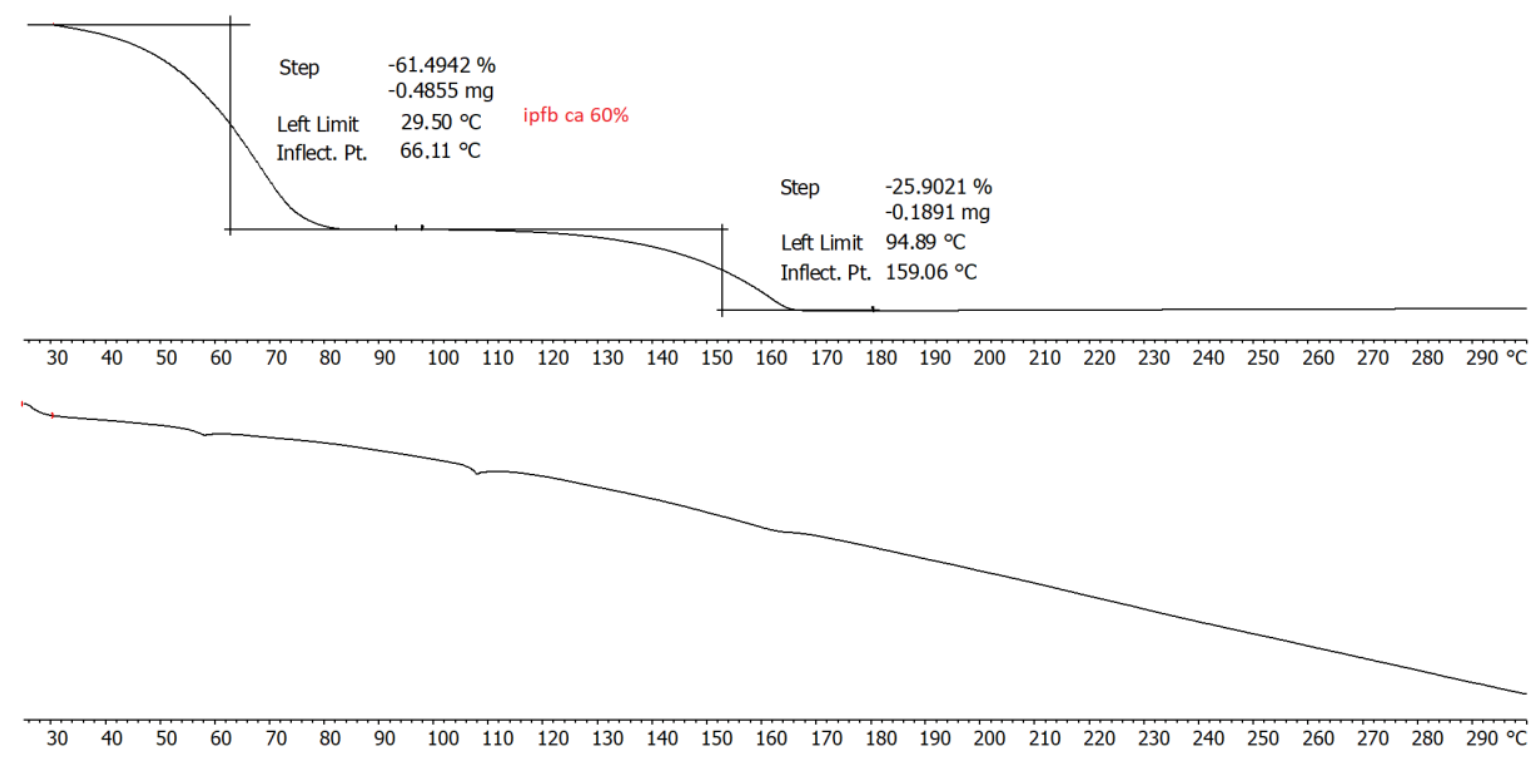

Figure S14. TG and DSC curves of the (ipfb)(pdon).

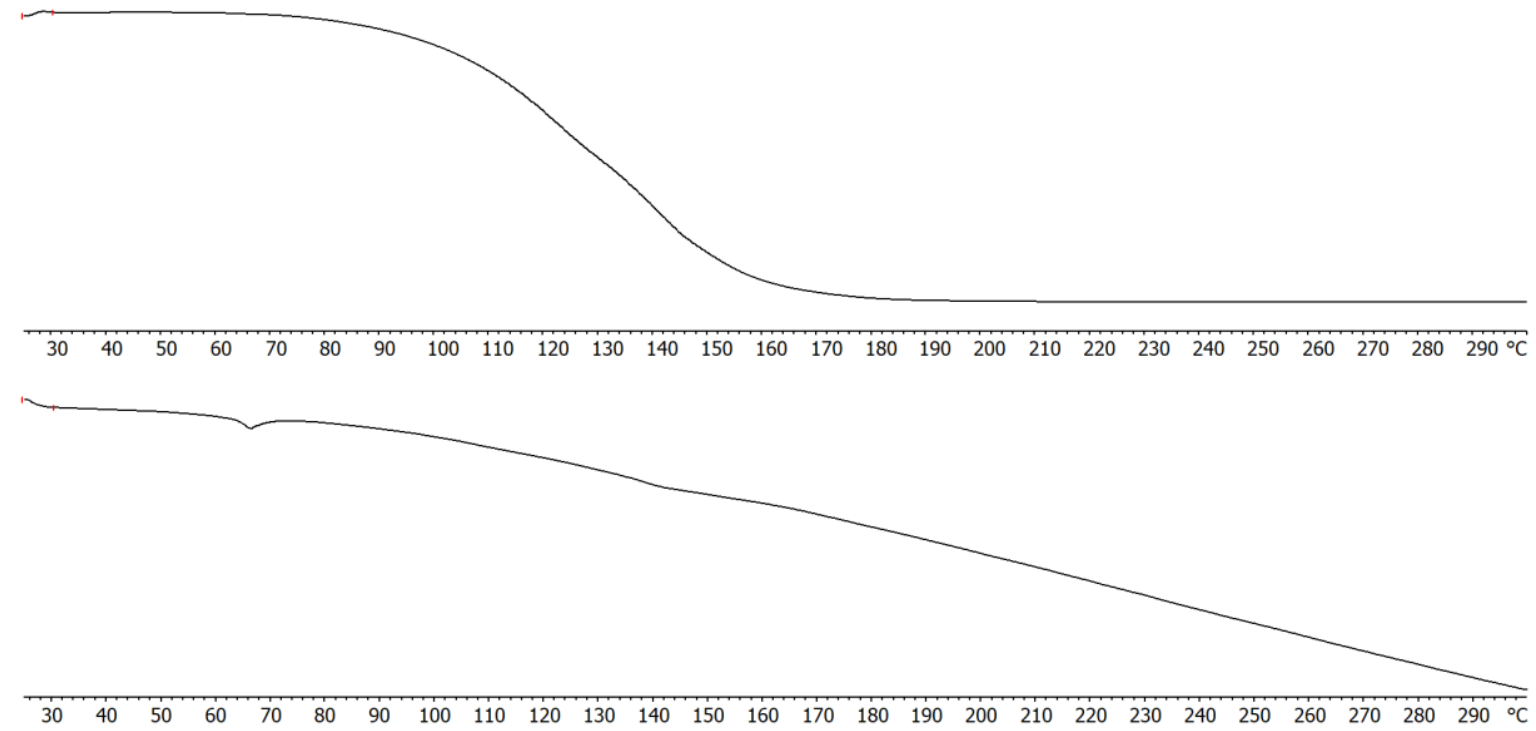

Figure S15. TG and DSC curves of the (12ditfb)(pdon)2. 


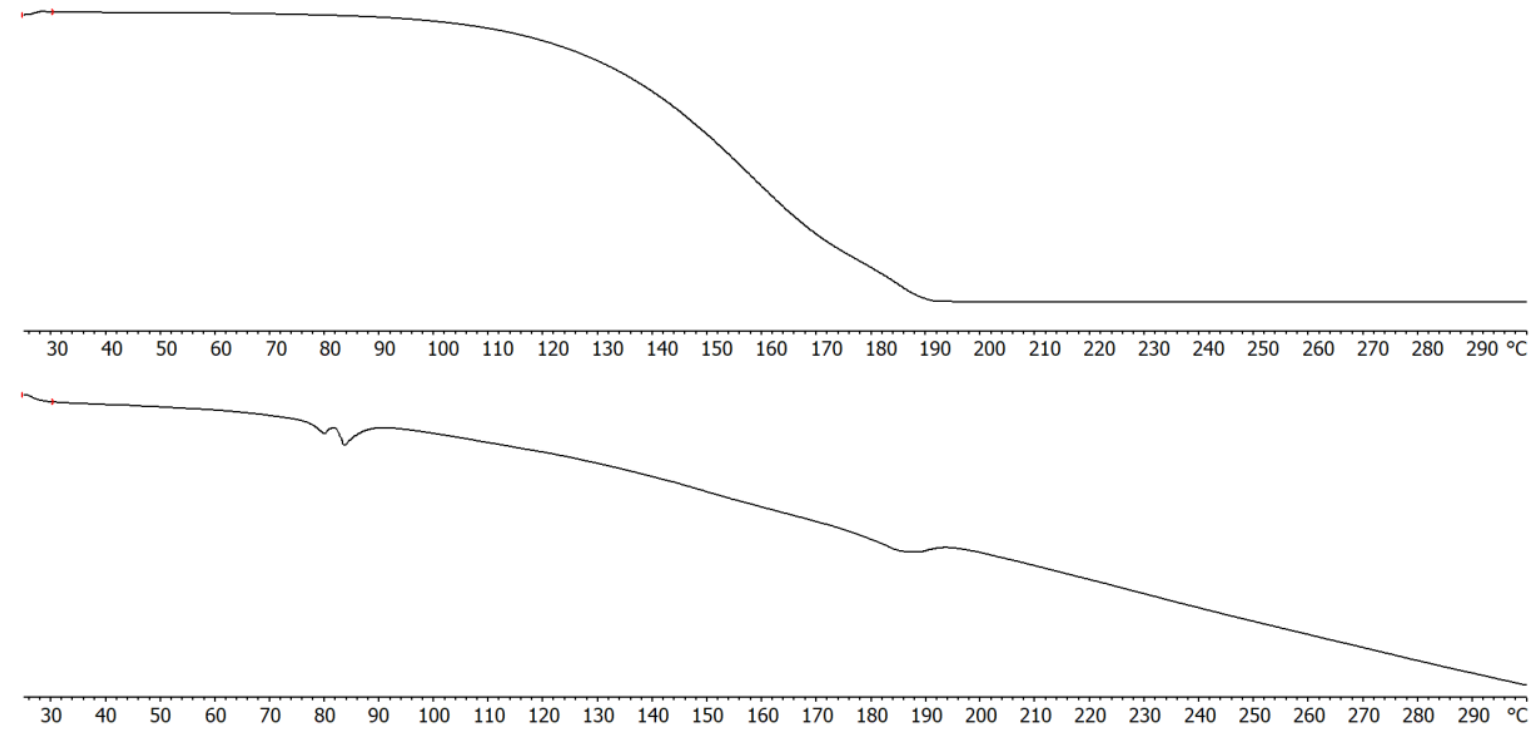

Figure S16. TG and DSC curves of the (13ditfb)(pdon)2.

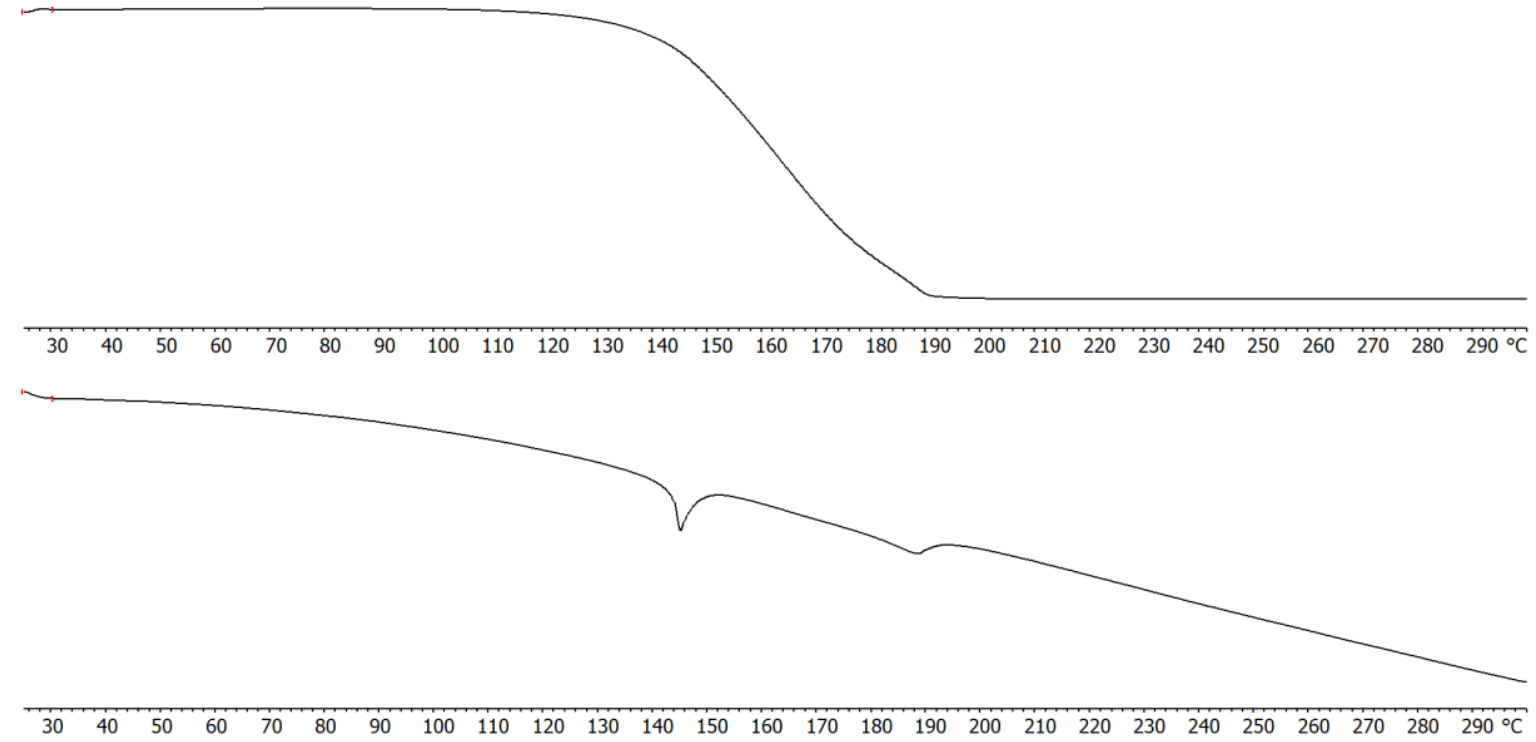

Figure S17. TG and DSC curves of the (14ditfb)(pdon)2. 


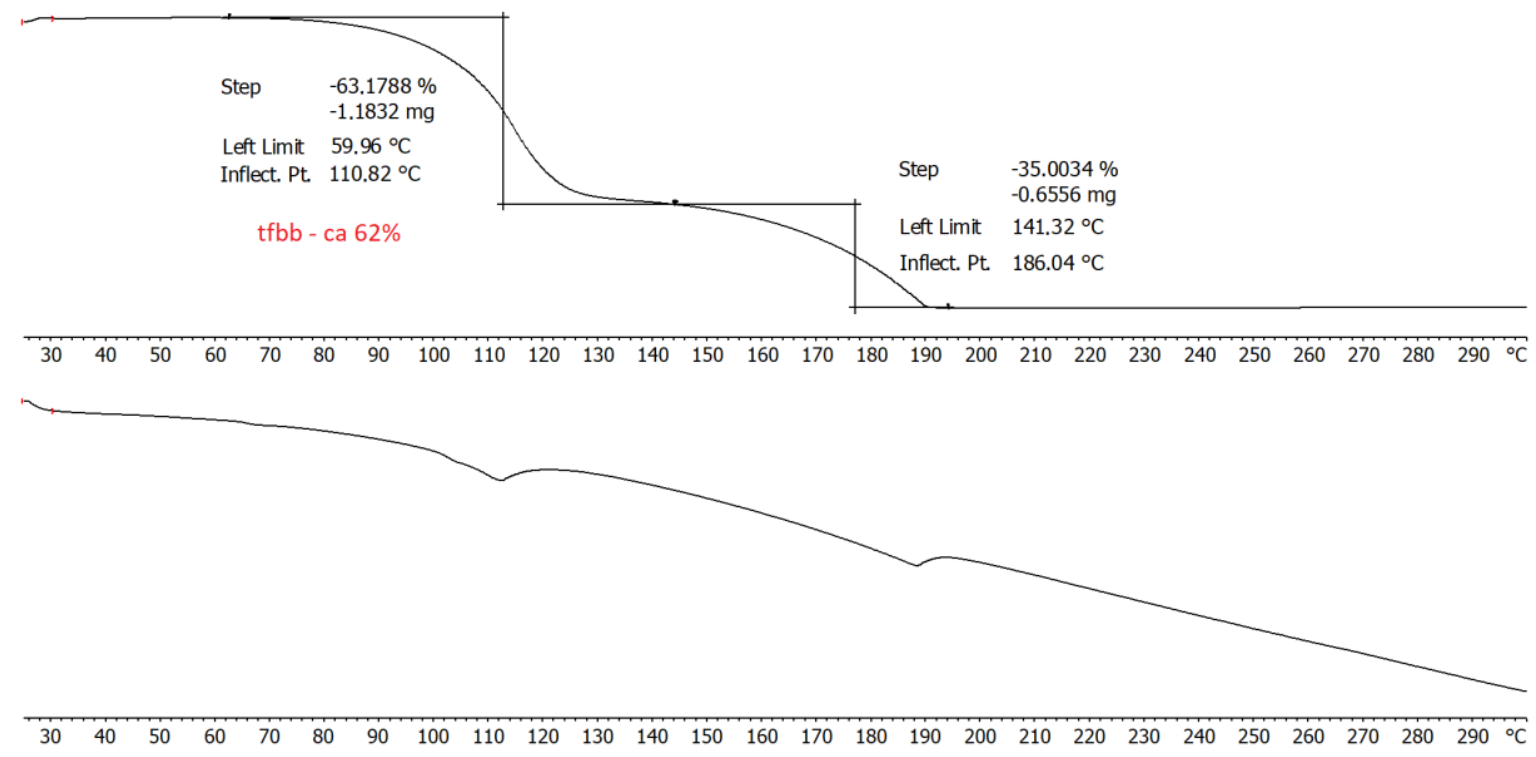

Figure S18. TG and DSC curves of the (13dbtfb)(pdon)2.

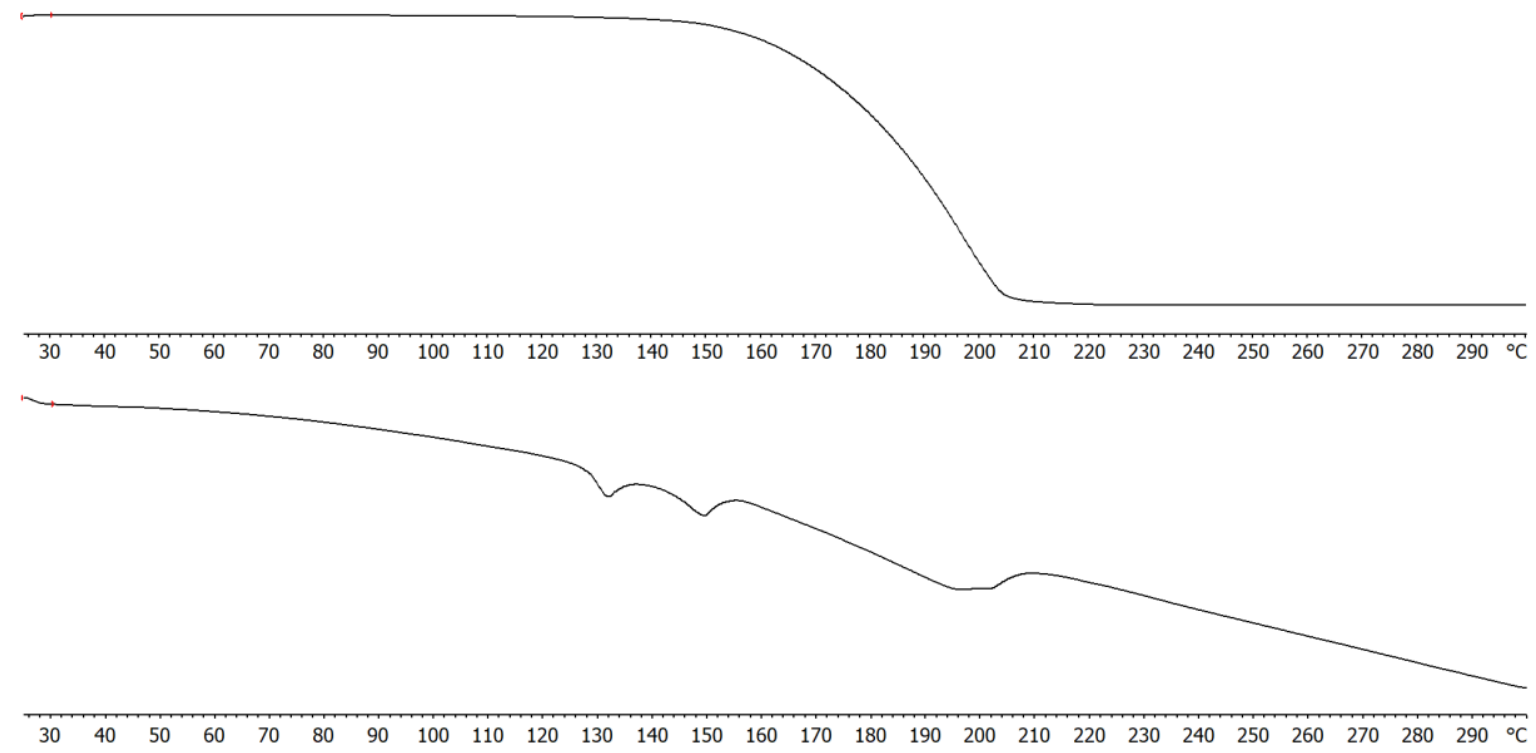

Figure S19. TG and DSC curves of the (135titfb)(pdon)2. 

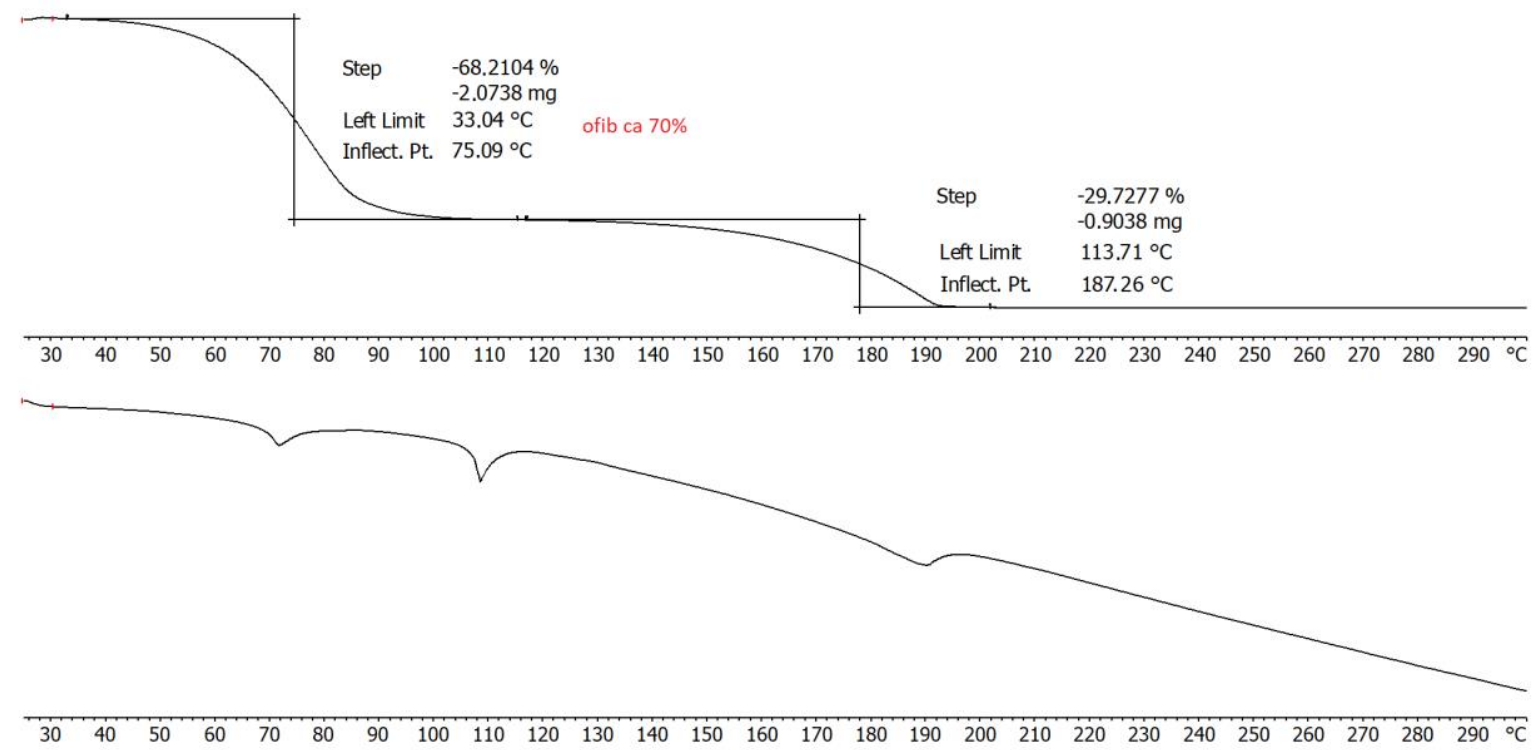

Figure S20. TG and DSC curves of the (ofib)(pdon)2. 


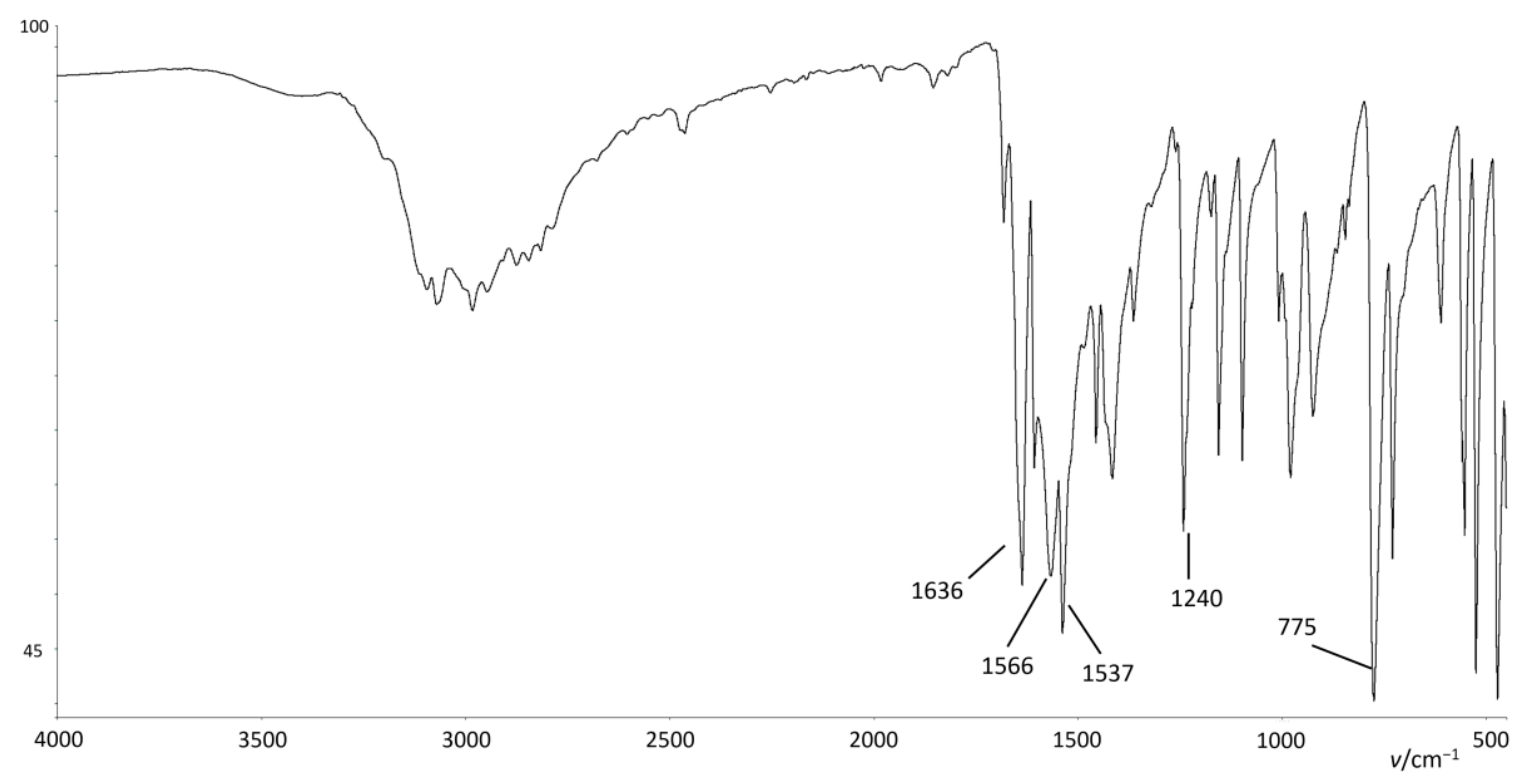

Figure S21. IR spectrum of the pdon.

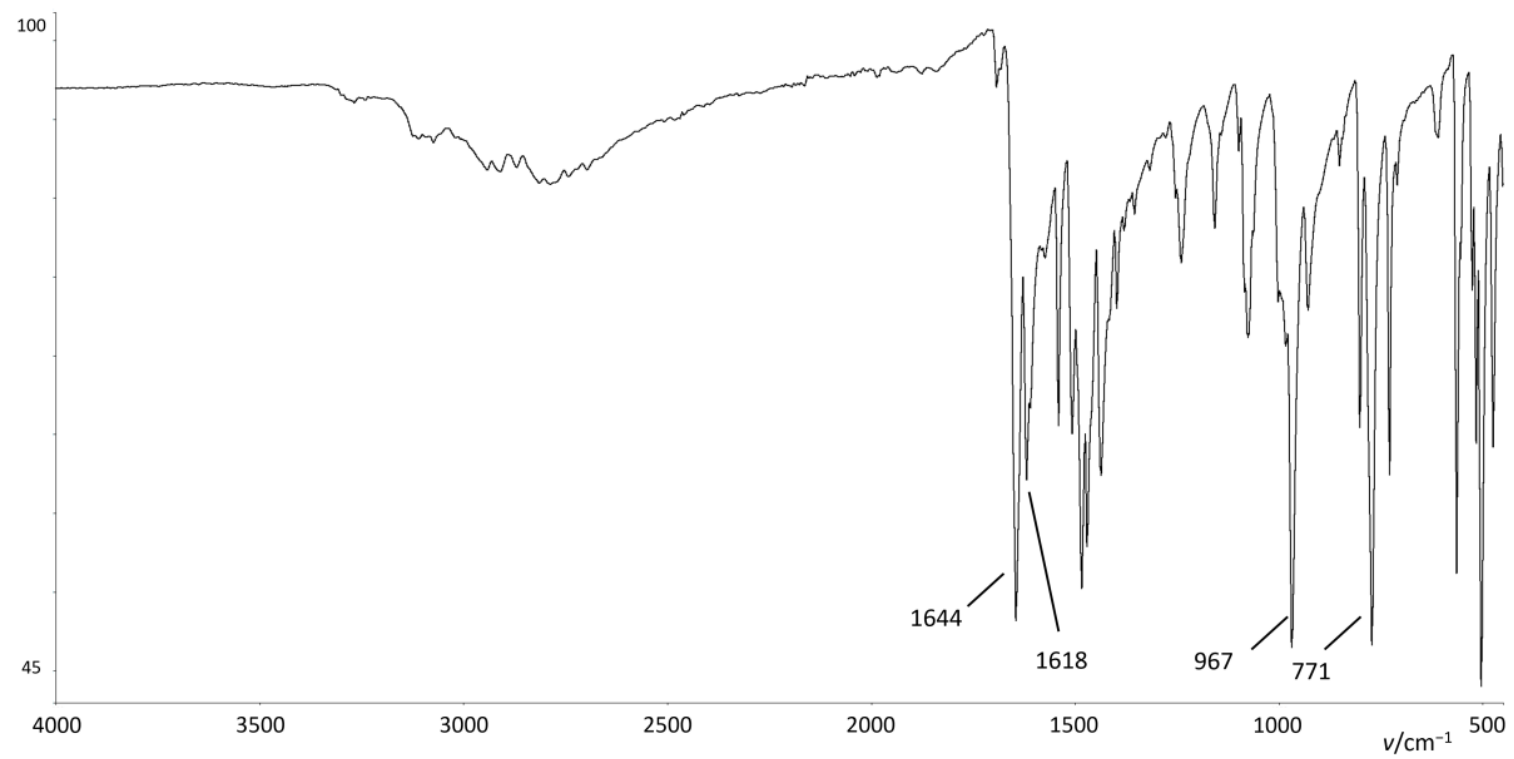

Figure S22. IR spectrum of the (ipfb)(pdon). 


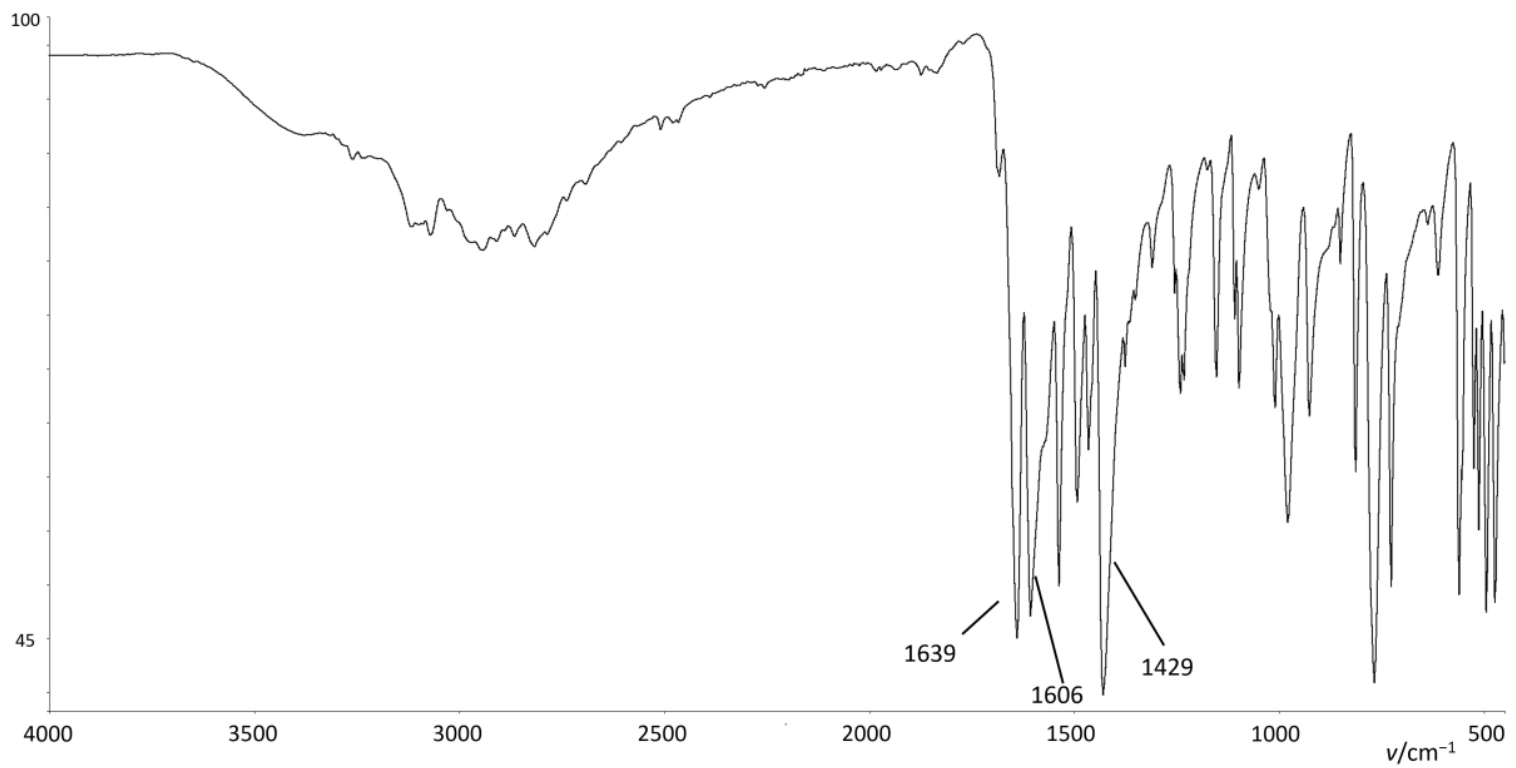

Figure S23. IR spectrum of the (12ditfb)(pdon) ${ }_{2}$.

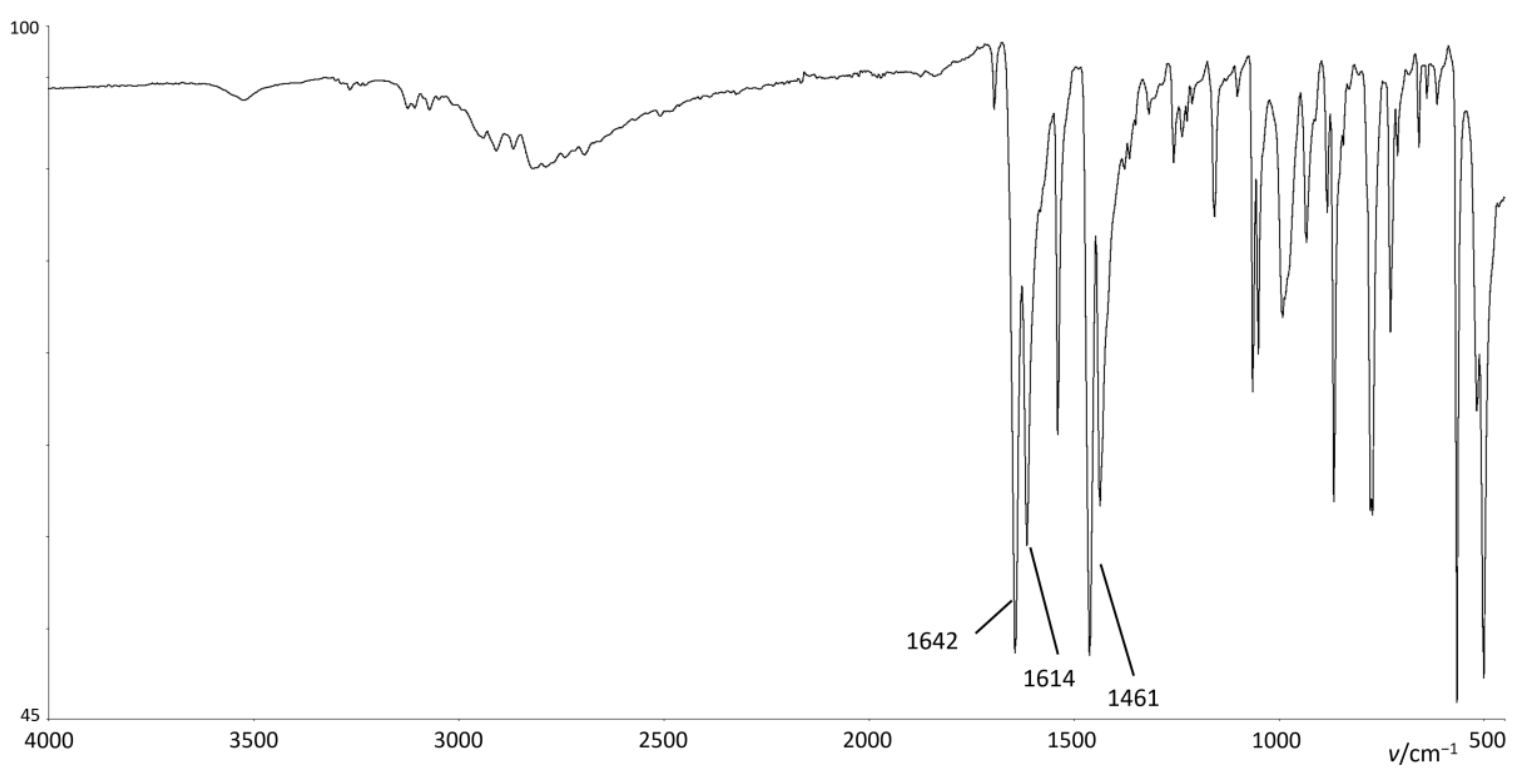

Figure S24. IR spectrum of the (13ditfb)(pdon)2. 


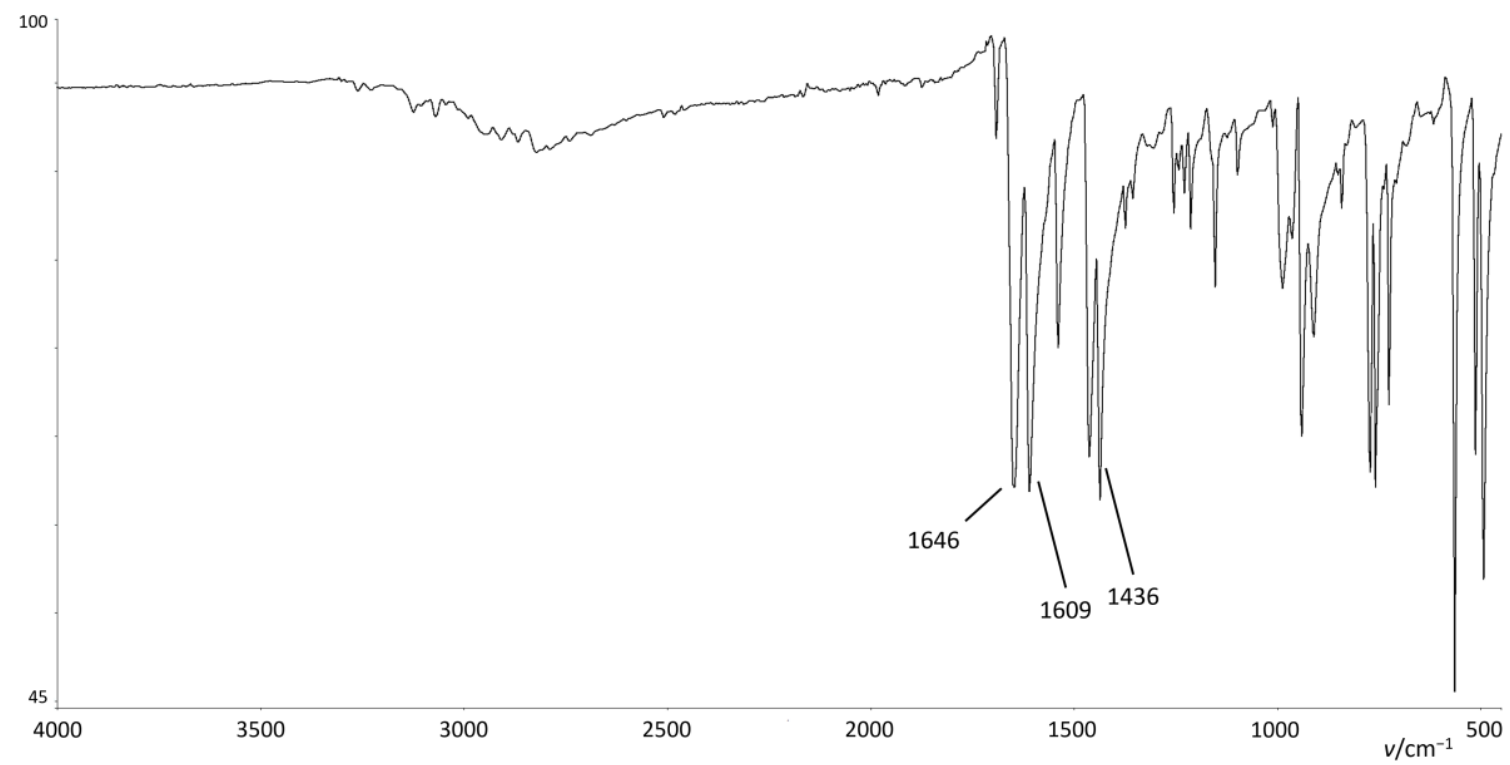

Figure S25. IR spectrum of the (14ditfb)(pdon) 2 .

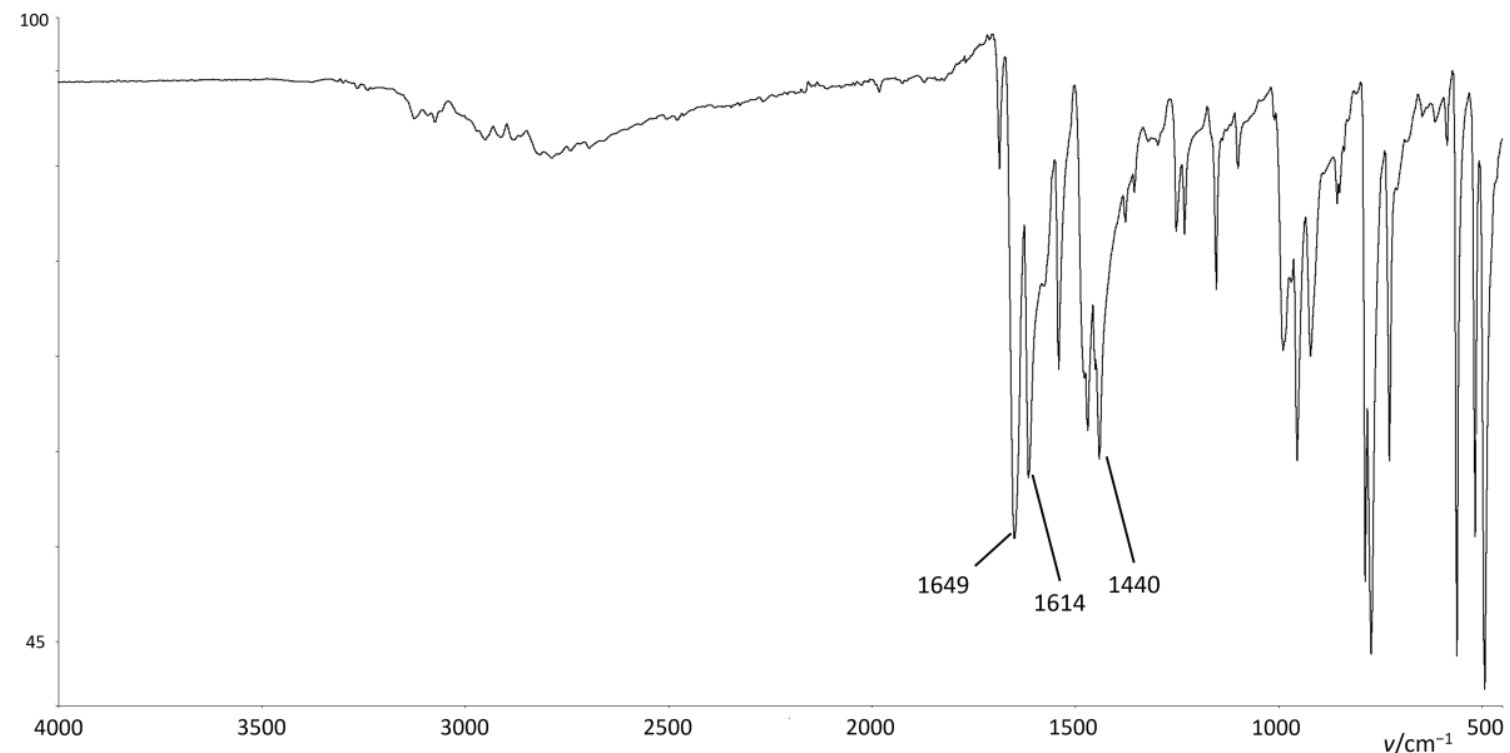

Figure S26. IR spectrum of the (14dbtfb)(pdon)2. 


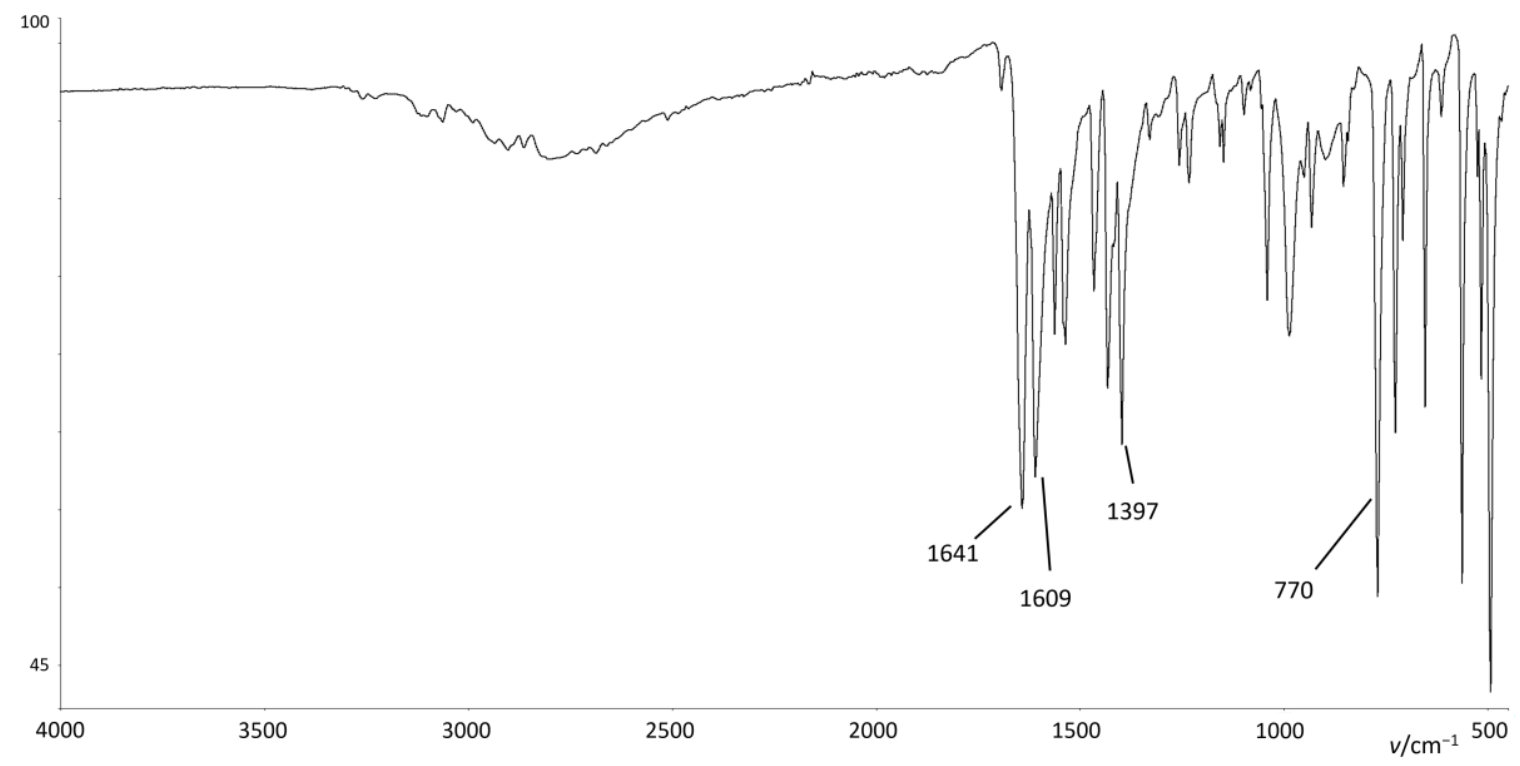

Figure S27. IR spectrum of the (135titfb)(pdon)2.

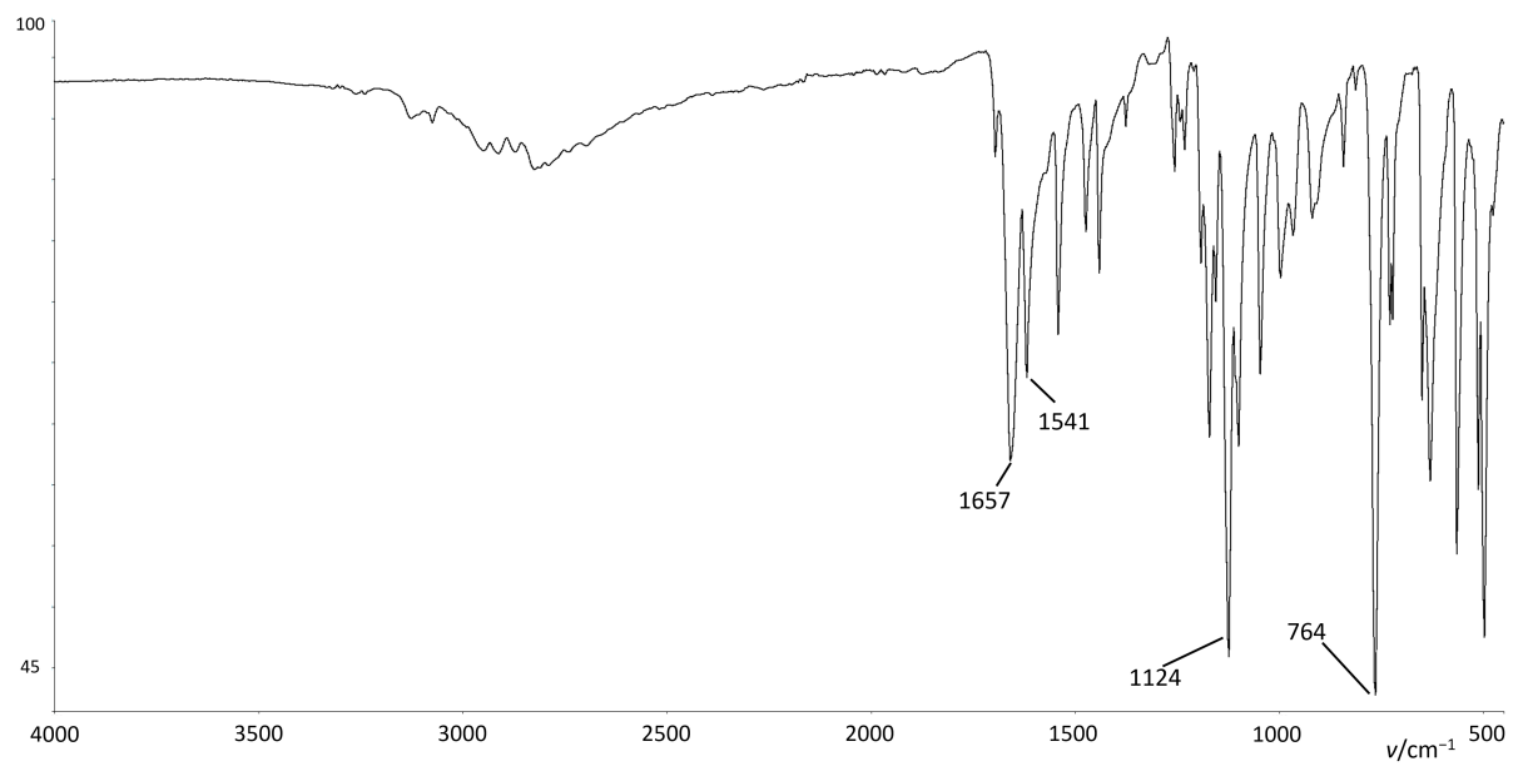

Figure S28. IR spectrum of the (ofib)(pdon)2. 
Table S1. An overview and crystallographic data of the prepared compounds.

\begin{tabular}{|c|c|c|c|}
\hline & (ipfb)(pdon) & $(12$ ditfb $)(\text { pdon })_{2}$ & $($ 13ditfb)(pdon) 2 \\
\hline Molecular formula & $\mathrm{C}_{11} \mathrm{H}_{5} \mathrm{NOF}_{5} \mathrm{I}$ & $\mathrm{C}_{16} \mathrm{H}_{10} \mathrm{~N}_{2} \mathrm{O}_{2} \mathrm{I}_{2} \mathrm{~F}_{4}$ & $\mathrm{C}_{16} \mathrm{H}_{10} \mathrm{~N}_{2} \mathrm{O}_{2} \mathrm{I}_{2} \mathrm{~F}_{4}$ \\
\hline$M r$ & 389.07 & 592.07 & 592.07 \\
\hline Crystal system & monoclinic & monoclinic & monoclinic \\
\hline Space group & $P 21 / c$ & $I 2 / a$ & $P 2_{1} / c$ \\
\hline$a / \AA$ & $7.4107(2)$ & $13.8467(5)$ & $10.8798(6)$ \\
\hline$b / \AA$ & $7.4217(1)$ & $11.4250(2)$ & $10.3443(4)$ \\
\hline$c / \AA$ & $22.9779(5)$ & $12.8921(5)$ & $16.8469(8)$ \\
\hline$\alpha /^{\circ}$ & 90 & 90 & 90 \\
\hline$\beta /^{\circ}$ & $96.657(2)$ & $118.083(4)$ & $100.921(5)$ \\
\hline$\gamma /{ }^{\circ}$ & 90 & 90 & 90 \\
\hline$V / \AA^{3}$ & $1255.26(3)$ & $1799.39(44)$ & 1861.68(18) \\
\hline Z & 4 & 4 & 4 \\
\hline$\rho_{\text {calc }} / \mathrm{g} \mathrm{cm}^{-3}$ & 2.03 & 2.19 & 2.11 \\
\hline $\mathrm{T} / \mathrm{K}$ & 170 & 170 & 170 \\
\hline$\mu / \mathrm{mm}^{-1}$ & 2.602 & 3.548 & 3.429 \\
\hline$F(000)$ & 716.0 & 1112.0 & 1112.0 \\
\hline $\begin{array}{l}\text { Refl. } \\
\text { collected/unique }\end{array}$ & $32945 / 2743$ & 14097 / 1972 & 31243 / 4063 \\
\hline parameters & 172 & 118 & 235 \\
\hline$\Delta \rho_{\max }, \Delta \rho_{\min } / \mathrm{e} \AA^{-3}$ & $5.882 ;-5.596$ & $0.530 ;-1.646$ & $3.300 ;-1.139$ \\
\hline$R\left[F^{2}>4 \sigma\left(F^{2}\right)\right]$ & 0.141 & 0.029 & 0.069 \\
\hline $\mathrm{w} R\left(F^{2}\right)$ & 0.253 & 0.073 & 0.150 \\
\hline Goodness of fit, $S$ & 1.080 & 1.168 & 1.052 \\
\hline
\end{tabular}

Table A1. Continuation 
Table S1. Continuation

\begin{tabular}{|c|c|c|c|}
\hline & $(14$ ditfb $)(\text { pdon })_{2}$ & $(14 d b t f b)(\text { pdon })_{2}$ & $(135$ titfb $)(\text { pdon })_{2}$ \\
\hline $\begin{array}{l}\text { Molecular } \\
\text { formula }\end{array}$ & $\mathrm{C}_{16} \mathrm{H}_{10} \mathrm{~N}_{2} \mathrm{O}_{2} \mathrm{I}_{2} \mathrm{~F}_{4}$ & $\mathrm{C}_{16} \mathrm{H}_{10} \mathrm{~N}_{2} \mathrm{O}_{2} \mathrm{Br}_{2} \mathrm{~F}_{4}$ & $\mathrm{C}_{16} \mathrm{H}_{10} \mathrm{~N}_{2} \mathrm{O}_{2} \mathrm{I}_{3} \mathrm{~F}_{3}$ \\
\hline$M r$ & 592.07 & 498.07 & 699.97 \\
\hline Crystal system & monoclinic & monoclinic & triclinic \\
\hline Space group & $P 21 / c$ & $P 21 / n$ & $P-1$ \\
\hline$a / \AA$ & $12.5097(3)$ & $12.4129(5)$ & $7.7093(2)$ \\
\hline$b / \AA$ & $8.1933(2)$ & $7.8380(3)$ & $10.8881(3)$ \\
\hline$c / \AA$ & $18.6002(4)$ & $18.2390(8)$ & $13.4276(4)$ \\
\hline$\alpha /^{\circ}$ & 90 & 90 & $68.054(2)$ \\
\hline$\beta /{ }^{\circ}$ & $106.459(3)$ & $107.876(5)$ & $84.115(2)$ \\
\hline$\gamma /{ }^{\circ}$ & 90 & 90 & $77.422(2)$ \\
\hline$V / \AA^{3}$ & 1828.32(17) & $1688.85(29)$ & $1020.07(10)$ \\
\hline Z & 4 & 8 & 2 \\
\hline$\rho_{\text {calc }} / \mathrm{g} \mathrm{cm}^{-3}$ & 2.15 & 1.96 & 2.28 \\
\hline$T / \mathrm{K}$ & 170 & 170 & 170 \\
\hline$\mu / \mathrm{mm}^{-1}$ & 3.492 & 4.855 & 4.635 \\
\hline$F(000)$ & 1112.0 & 968.0 & 644.0 \\
\hline $\begin{array}{l}\text { Refl. } \\
\text { collected/uniq } \\
\text { ue }\end{array}$ & 29682 / 3985 & 14483 / 3305 & 16558 / 4448 \\
\hline parameters & 235 & 235 & 235 \\
\hline $\begin{array}{c}\Delta \rho \max , \Delta \rho \min / \mathrm{e} \\
\AA^{-3}\end{array}$ & $0.883 ;-2.068$ & $1.200 ;-1.882$ & $1.319 ;-1.090$ \\
\hline$R\left[F^{2}>4 \sigma\left(F^{2}\right)\right]$ & 0.042 & 0.062 & 0.046 \\
\hline $\mathrm{w} R\left(F^{2}\right)$ & 0.112 & 0.171 & 0.118 \\
\hline $\begin{array}{l}\text { Goodness of } \\
\text { fit, } S\end{array}$ & 1.155 & 1.053 & 1.043 \\
\hline
\end{tabular}


Table S1. Continuation

\begin{tabular}{|c|c|}
\hline & $($ ofib $)(\text { pdon })_{2}$ \\
\hline Molecular formula & $\mathrm{C}_{14} \mathrm{H}_{10} \mathrm{~N}_{2} \mathrm{O}_{2} \mathrm{I}_{2} \mathrm{~F}_{8}$ \\
\hline$M r$ & 643.88 \\
\hline Crystal system & monoclinic \\
\hline Space group & $C 2 / c$ \\
\hline$a / \AA$ & $27.4114(6)$ \\
\hline$b / \AA$ & $7.0340(2)$ \\
\hline$c / \AA$ & $20.2776(5)$ \\
\hline$\alpha /^{\circ}$ & 90 \\
\hline$\beta /{ }^{\circ}$ & $98.227(2)$ \\
\hline$\gamma /{ }^{\circ}$ & 90 \\
\hline$V / \AA^{3}$ & $3869.52(11)$ \\
\hline Z & 8 \\
\hline$\rho_{\text {calc }} / \mathrm{g} \mathrm{cm}^{-3}$ & 2.21 \\
\hline$T / \mathrm{K}$ & 170 \\
\hline$\mu / \mathrm{mm}^{-1}$ & 3.338 \\
\hline$F(000)$ & 2416.0 \\
\hline $\begin{array}{c}\text { Refl. } \\
\text { collected/unique }\end{array}$ & 35205 / 5641 \\
\hline parameters & 253 \\
\hline$\Delta \rho_{\max }, \Delta \rho_{\min } / \mathrm{e} \AA^{-3}$ & $1.206 ;-0.746$ \\
\hline$R\left[F^{2}>4 \sigma\left(F^{2}\right)\right]$ & 0.034 \\
\hline $\mathrm{w} R\left(F^{2}\right)$ & 0.069 \\
\hline Goodness of fit, $S$ & 1.072 \\
\hline
\end{tabular}


Table S2. Halogen bond lengths and their relative shortenings (rs) in obtained compounds.

\begin{tabular}{|c|c|c|c|}
\hline compound & $d(\mathrm{I} \cdots \mathrm{O}) / \AA$ & $d-\mathrm{r}_{\mathrm{vdw}} / \AA$ & $r s / \%$ \\
\hline$($ 12ditfb $)(\text { pdon })_{2}$ & 3.041 & -0.459 & 13.1 \\
\hline \multirow{2}{*}{$(13 \operatorname{ditfb})(\text { pdon })_{2}$} & 2.765 & -0.735 & 21.0 \\
\hline & 2.817 & -0.683 & 19.5 \\
\hline \multirow{2}{*}{$(14 \operatorname{ditfb})(\text { pdon })_{2}$} & 2.795 & -0.705 & 20.1 \\
\hline & 2.807 & -0.693 & 19.8 \\
\hline \multirow{3}{*}{$($ 135ditfb $)(\text { pdon })_{2}$} & 2.840 & -0.660 & 18.9 \\
\hline & 2.920 & -0.580 & 16.6 \\
\hline & 2.941 & -0.559 & 16.0 \\
\hline \multirow{2}{*}{$(14 \mathrm{dbtfb})(\text { pdon })_{2}$} & 2.876 & -0.494 & 14.7 \\
\hline & 2.925 & -0.445 & 13.2 \\
\hline$(\mathrm{ipfb})$ (pdon) & 2.698 & -0.802 & 22.9 \\
\hline \multirow{2}{*}{$($ ofib $)(\text { pdon })_{2}$} & 2.775 & -0.725 & 20.7 \\
\hline & 2.789 & -0.711 & 20.3 \\
\hline
\end{tabular}




\section{Preparation of the cocrystals}

Cocrystals were prepared by dissolving pdon $(10 \mathrm{mg}, 105.1 \mathrm{mmol})$ and corresponding halogen bond donor (Table 2 ) in $3 \mathrm{~mL}$ of hot acetone/ethanol mixture (1:1). Single crystals suitable for X-ray structure determination were obtained after cooling to the room temperature (ca. $25^{\circ} \mathrm{C}$ ).

Table S2. Masses and volumes of the halogen bond donors.

\begin{tabular}{|c|c|}
\hline XB donor & mass / volume \\
\hline ipfb & $16 \mu \mathrm{L}$ \\
\hline $12 \mathrm{ditfb}$ & $89 \mathrm{mg}$ \\
\hline $13 \mathrm{ditfb}$ & $31 \mu \mathrm{L}$ \\
\hline $14 \mathrm{ditfb}$ & $89 \mathrm{mg}$ \\
\hline $14 \mathrm{dbtfb}$ & $68 \mathrm{mg}$ \\
\hline $135 \mathrm{titfb}$ & $166 \mathrm{mg}$ \\
\hline ofib & $41 \mu \mathrm{L}$ \\
\hline
\end{tabular}

\section{X-Ray Diffraction Measurements}

All single crystal X-ray diffraction experiments were performed using an Oxford Diffraction XtaLAB Synergy, Dualflex, HyPix Xray four-circle diffractometer with mirror-monochromated $\operatorname{MoK} \alpha(\lambda=0.71073 \AA)$ radiation. The data sets were collected using the $\omega$-scan mode over the $2 \theta$-range up to $60^{\circ}$. Programs CrysAlis PRO CCD and CrysAlis PRO RED were employed for data collection, cell refinement, and data reduction.[1, 2] The structures were solved by SHELXT or by direct methods using the SHELXS and refined using SHELXL programs.[3, 4] The structural refinement was performed on F2 using all data. The hydrogen atoms were placed in calculated positions and treated as riding on their parent atoms $[\mathrm{C}-\mathrm{H}=$ $0.93 \AA$ and $\operatorname{Uiso}(\mathrm{H})=1.2 \operatorname{Ueq}(\mathrm{C}) ; \mathrm{C}-\mathrm{H}=0.97 \AA$ and $\operatorname{Uiso}(\mathrm{H})=1.2 \mathrm{Ueq}(\mathrm{C})]$. All calculations were performed using the WinGX or Olex2 1.3-ac4 crystallographic suite of programs.[5] Further details are available from the Cambridge Crystallographic Centre. Molecular structures of compounds and their packing diagrams were prepared using Mercury.[6]

\section{Thermal analysis}

Differential scanning calorimetry (DSC) and thermogravimetric (TG) measurements were performed simultaneously on a Mettler-Toledo TGA/DSC 3+ module (Mettler Toledo, Greifensee, Switzerland). Samples were placed in alumina crucibles $(40 \mu \mathrm{L})$ 
and heated 25 to $300{ }^{\circ} \mathrm{C}$, at a heating rate of $10^{\circ} \mathrm{C}$ min- 1 under nitrogen flow of 150 mL min-1.

Data collection and analysis were performed using the program package STARe Software (Version 15.00, Mettler Toledo, Greifensee, Switzerland).[7] TG and DSC thermograms of the prepared compounds are shown in Figures S11-S15 in Supplementary Materials.

\section{References:}

(1) Manual, U. CrysAlis Pro. Power. Agilent Technologies Ltd: Yarnton, Oxfordshire, England 2014.

(2) CrysAlis PRO CCD. Agilent Technologies Ltd: Yarnton, Oxfordshire, England 2014.

(3) Sheldrick, G. M. Crystal Structure Refinement with SHELXL. Acta

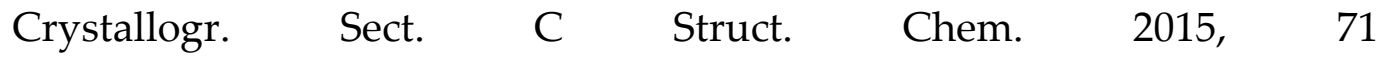
https://doi.org/10.1107/S2053229614024218.

(4) Sheldrick, G. M. SHELXT - Integrated Space-Group and Crystal-Structure Determination. Acta Crystallogr. Sect. A Found. Adv. 2015, 71 (1). https://doi.org/10.1107/S2053273314026370.

(5) Farrugia, L. J. WinGX Suite for Small-Molecule Single-Crystal Crystallography. J. Appl. Crystallogr. 1999, $32 \quad$ (4). https://doi.org/10.1107/S0021889899006020.

(6) Macrae, C. F.; Bruno, I. J.; Chisholm, J. A.; Edgington, P. R.; McCabe, P.; Pidcock, E.; Rodriguez-Monge, L.; Taylor, R.; van de Streek, J.; Wood, P. A. Mercury CSD 2.0 - New Features for the Visualization and Investigation of Crystal Structures. J. Appl. Crystallogr. 2008, 41 (2). https://doi.org/10.1107/S0021889807067908.

(7) STARe Software. Mettler Toledo: Greifensee, Switzerland 2016. 